\title{
Validación del cuestionario de optimismo disposicional usando la teoría de respuesta al ítem*
}

\section{Validation of questionnaire dispositional optimism using item response theory}

\author{
Marcela Velasco Salamanca \\ Constanza Londoño Pérez** \\ Ivonne Alejo Castañeda \\ Universidad Católica de Colombia \\ Recibido: 2 de octubre de 2013 \\ Revisado: 30 de noviembre de 2013 \\ Aceptado: 3 de febrero de 2014
}

\section{Resumen}

La presente investigación tenía por objetivo establecer las condiciones psicométricas de validez y confiabilidad del Cuestionario de Optimismo Disposicional, se aplicó a una muestra incidental de 608 personas (16 a 60 años), nivel educativo diverso, de ambos géneros y residentes en Bogotá. La validación de contenido se hizo con metodología ANGOFF, la validación concurrente con el Test de orientación vital LOT y el análisis de confiabilidad con el modelo de Rasch. Se concluye que la prueba cuenta con una confiabilidad adecuada, está ajustada y cumple criterios de calidad psicométrica, teniendo en cuenta que aunque el Alpha de Cronbach no es tan elevado, el nivel de separación reportado desde el modelo de Rasch es el esperado, un nivel de ajuste apropiado.

Palabras clave: optimismo, pesimismo, validez, confiabilidad, consistencia. 


\section{Abstract}

The aim of this research was to find the psychometric qualities of validity and reliability of the Dispositional Optimism Questionnaire, with a convenience sample of 608 participants aged 16 to 60 , from diverse educational backgrounds, both genders, and residing in Bogota, Colombia. The content validity was performed with an ANGOFF methodology, the concurrent validity with the Life Orientation Test (LOT) and the reliability analysis with the Rasch model. The test was found to be reliable and psychometrically adequate. Even though the Cronbach's alpha was not so high, the separation reported by the Rasch model was expected, an appropriate fit.

Keywords: Optimism, pessimism, validity, reliability, consistency.

En la actualidad se ha dado especial énfasis a la psicología positiva, que surge como respuesta ante la mirada negativa de una psicología que se había centrado casi exclusivamente en el estudio de fenómenos psicopatológicos más que en las fortalezas de los individuos como factor de afrontamiento de eventos estresantes (Vera, 2006). Esta rama reciente de la psicología busca comprender los procesos que subyacen a las cualidades positivas del ser humano, entre los que se cuentan la alegría, la satisfacción, la felicidad y el optimismo; a la vez que estudia la variación entre individuos y culturas de dichas cualidades, las cuales no han sido suficientemente abordadas (Hartmann, Chneider y Emrich, 2003).

La investigación realizada por Alpízar y Salas (2010), demostró que personas con características positivas tienden a desarrollar habilidades y fortalezas en respuesta a diversas situaciones cotidianas, además buscan activamente la felicidad; las actitudes positivas son considerados rasgos de personalidad que se reflejan en la disposición a reconocer los aspectos beneficiosos de los eventos, en la seguridad que tiene la persona sobre la alta probabilidad de ocurrencia de eventos buenos y positivos en su vida, en el mejor afrontamiento de las situaciones, y en la vivencia de menores niveles de estrés y ansiedad.

En general, se considera que el bienestar tiene una dimensión básica que es subjetiva, con dos componentes, uno afectivo-emocional y otro relacionado con los aspectos cognitivos, ambos aso- ciados a la evaluación de la satisfacción que hace la persona de su propia vida (Andrews y Withey, 1976; Diener y Suh, 2000; Diener, Suh, Lucas y Smith, 1999). El psicólogo no solo tendría un interés en contribuir a la reducción del malestar psicológico, sino que su labor estaría encaminada más allá de lo estrictamente psicopatológico (Salas, 2008; Seligman, 2003).

Los estudios sobre optimismo tienen su origen en la reformulación teórica de la propuesta de indefensión aprendida de Abramson, Seligman y Teasdale (1978), en la que se identifica que este puede ser abordado desde dos perspectivas teóricas complementarias: el Estilo Explicativo Pesimista-Optimista de Peterson y Seligman (1984), en la que los individuos muestran alta tendencia a manifestar que los sucesos negativos que les ocurren se deben a factores internos propios, que son estables a través del tiempo y que tiene efecto en la vida de todas las personas; y en forma contraria el optimismo disposicional de Scheier y Carver (1985; 2014), quienes lo consideran como la postura de esperar resultados favorables en todos los ámbitos de la vida.

En esta misma línea Ji, Zhang, Usborne y Guan (2004); Lai y Yue (2000) y Londoño (2009), aportan que el optimismo es considerado como el conjunto de expectativas favorables generalizadas en relación con los eventos que afronta una persona en la vida y proponen que este es un constructo unidimensional polar; por tanto, esto implica que el optimismo es un opuesto directo del pesimismo y 
que ambos son rasgos relativamente estables, consistentes a través del tiempo y las situaciones. Así, en el pesimismo las causas atribuidas o explicativas de los eventos son vistas como internas (estilo atribucional interno), permanentes en el tiempo independientemente del contexto y que genera efectos globales en la vida de las personas.

En oposición el estilo optimista es definido por Anadón-Revuelta (2006), Marrero y Carballeira (2010), como la tendencia del individuo a explicar los sucesos negativos a partir de una causa externa (estilo atribucional externo), el cual es variable en el tiempo y solo se remite a una situación específica no perdurable. Para unos autores corresponde a una dimensión aprendida de la personalidad, producto de la interacción herenciaambiente; y para otros como es una característica disposicional de personalidad que media entre los acontecimientos externos y la interpretación personal de los mismos (Kim, Seidlitz, Ro, Evinger y Duberstein, 2004; Seligman, 2005).

El optimista está motivado por la creencia de que los resultados deseados son fácilmente alcanzables con los recursos de los que dispone, y logra sus metas porque posee alta autonomía y autocontrol que le facilitan cumplir sus objetivos personales (Londoño, 2009). Este individuo asume que si no se han alcanzado los objetivos trazados seguramente lo logrará en un futuro inmediato con algo de esfuerzo programado, condición que redunda en la calidad de vida percibida de las personas enfermas y en la recuperación misma (Begoña, Panduro, Crespillo, Rojas y González, 2010; Kennedy y Hughes, 2004; Morales, Arenas, Reig-Ferrer, Álvarez, Malek, Moledous y Cotilla, 201; Naghi, Philip, Cleenewerck y Schwarz, 2012; Ortiz, Ramos y Vera-Villarroel, 2003).

Estudios realizados por Chico (2002), señalan que esta estrategia de afrontamiento contribuye a que las personas toleren de manera adecuada períodos estresantes; el segundo tipo es el optimismo a ultranza, que consiste en que la persona puede incurrir en no tomar precauciones, ser negligente al percibir un grado de vulnerabilidad muy bajo respecto a diferentes riesgos. Dentro de este pueden citarse dos tipos: el optimismo defensivo o ingenuo, que es una clara tendencia del individuo a encubrir los peligros verdaderos creyendo que no se está corriendo riesgo (invulnerabilidad), y el optimismo funcional, el cual se traduce en conductas de hipervigilancia acompañadas de conductas protectoras en ocasiones exageradas (tendencias paranoides) (Vera-Villarroel, CórdovaRubio y Celis-Atenas, 2009).

Cuando el optimismo de la persona disposicional y realista incrementa su potencial de recursos para adaptarse y responder ante diversas enfermedades ya que retarda el deterioro, aumenta el tiempo de sobrevida e incrementa la funcionalidad (Gison, Dall'armi, Donati, Rizza y Guiaquinto, 2014; Oreskovic y Goodman, 2013; Senkus, Cardoso y Pagani, 2014). La gente optimista no solo tiene mejor ajuste ante el estrés, además incorpora comportamientos saludables más fácilmente que los individuos no optimistas (Black y Reynolds, 2011). El efecto positivo del optimismo disposicional sobre la salud y la adaptación efectiva a múltiples situaciones que ponen a prueba los recursos de los individuos, ha hecho evidente la importancia de contar con medidas objetivas y confiables que permitan identificar si una persona es optimista, y si esta característica es estable en diversas situaciones. Esto para diferenciar si dicho optimismo corresponde más a una visión positiva ingenua, o al optimismo realista que se caracteriza por combinar altas expectativas de buen resultado, y la disposición de los recursos personales hacia el despliegue de una cadena de conductas para hacer frente a las situaciones estresantes con la confianza de salir adelante (Londoño, Hernández, Alejo y Pulido, 2013).

Una de las pruebas diseñadas para evaluar el optimismo es el Test de Orientación Vital (Life Orientation Test -LOT-), cuya versión disponible es la revisada por Scheier, Carver y Bridges en 1994 (LOT-R), que consta de 10 ítems en escala Likert de 5 puntos, 6 ítems pretenden medir la dimensión de optimismo disposicional, en tanto que los otros 4 ítems son distractores o de "relleno" denominados así por el autor. De los 6 ítems, 3 están redactados en sentido positivo (dirección optimista) y 3 en sentido negativo (dirección pesimista) (Scheier, Carver y Bridges, 1994). 
Diversos estudios han analizado las propiedades psicométricas del LOT-R obteniendo resultados que parecen demostrar su confiabilidad y validez, basados en la medida del Alpha de Cronbach y en el análisis de factores (Heinonen, Raikkonen, Matthews, Scheier, Raitakari, Pulkki y KeltikangasJarvinen, 2006; Laranjeira, 2008; Vera-Villarroel, Córdova-Rubio y Celis-Atenas, 2009), no obstante, existen varias dificultades que no pueden perderse de vista, en primer lugar el hecho de que sean solo 6 ítems limita la posibilidad de validar qué tan estable y permanente es el tipo de creencia positiva o negativa ante diversas situaciones de la vida, incluso si esta no corresponde más bien a una tendencia optimista no realista que ha sido claramente asociada a menor salud y menor probabilidad de recuperación de la salud en casos de enfermedad.

En segundo lugar, el número reducido de ítems aplicados en poblaciones grandes puede llevar a un error de apreciación por los índices de correlación como el Alpha de Cronbach (Brown, 1999); y tercero, los factores se ven claramente diferenciados probablemente debido a que cada uno incluye únicamente tres preguntas. Adicionalmente, algunos resultados acerca de la prueba son inconsistentes a través de varias culturas (Ji et al. 2004; Lai y Yue, 2000), situación que podría explicarse tanto desde la perspectiva de las diferencias culturales como de la perspectiva de la extensión de la prueba, o a la excesiva homogeneidad de las muestras (Pere, Chico y Tous, 2002).

Existe además la Escala de Optimismo Disposicional/Pesimismo -EOP-, diseñada por Londoño et al. (2013), constituida por 20 ítems que evalúan la tendencia personal hacia el optimismo disposicional. El cuestionario incluye distintas situaciones cotidianas que se dan en las distintas áreas de la vida de las personas e incluye tres opciones de respuesta: optimismo, sesgo optimista y pesimismo. Se han realizado distintas aplicaciones en poblaciones diversas (Londoño, 2009; Londoño, Alejo, Velasco y Botero, 2013; Velasco y Londoño, 2009) con índices de consistencia que oscilaban entre el 0.85 y 0.95 ; no obstante, Gómez y Londoño (2012), aplicaron el instrumento a 200 adultos y notaron que el índice de consistencia descendió a un nivel moderado bajo 0.53 en población adulta.
De otra parte el Modelo de Rash, específicamente la Teoría de la Respuesta al Ítem - TRI-, es un diseño teórico que busca explicar el comportamiento de datos empíricos como resultado de la aplicación de un instrumento psicométrico, mediante el uso de herramientas estadísticas y matemáticas para evaluar la calidad técnica de cada uno de los ítems o reactivos por separado, del instrumento como un todo y a la vez estimar el nivel que cada examinado presenta ante la prueba. Dentro de las ventajas ofrecidas por la TRI se encuentra que puede ser un modelo complementario a la Teoría Clásica (TC), que permite estimar mediciones psicológicas adicionales como las características de cada ítem y la precisión en la estimación de las puntuaciones de los sujetos (Tomás, González y Gómez, 2000).

Teniendo en cuenta la importancia de contar con medidas de comprobada fiabilidad y validez, y considerando que el diseño y validación de una prueba es un proceso complejo, el presente estudio tiene como pretensión reconfirmar los hallazgos acerca de las condiciones psicométricas del instrumento a través de la aplicación del modelo de Rasch, una vez se realizó una revisión de los ítems y de las opciones de respuesta. Así, esta investigación se centra en responder la pregunta: ¿cuáles son las condiciones psicométricas de validez y confiabilidad del Cuestionario de Optimismo Disposicional diseñado por Londoño, Hernández, Alejo \&yPulido, (2013) en la versión para adultos y jóvenes, usando la TRI?

\section{Método}

El presente estudio corresponde a una investigación de corte psicométrico, cuyas pretensiones se centran en analizar las condiciones psicométricas (validez, confiabilidad) de un instrumento, al igual que detallar su forma de aplicación, calificación e interpretación de la misma.

\section{Participantes}

La muestra no probabilística estuvo conformada por 615 personas con la estrategia bola de nieve, pero se excluyeron 7 casos teniendo en cuenta 
que la omisión de respuestas en el Cuestionario de Optimismo sobrepasó el $5 \%$ de los ítems, por lo cual la muestra final para el análisis fue de 608 personas: 336 mujeres y 272 hombres cuyas edades oscilaban entre 15 y 60 años de edad, con una media de 23.3 años, de estratos 0 a 5 , equilibrada entre distintos niveles de educación, así: $29.9 \%$ bachilleres, $27.5 \%$ técnicos, $24.2 \%$ profesionales y $18.4 \%$ especialización o maestría.

\section{Instrumentos}

Life Orientation Test -LOT-, versión revisada por Scheier, Carver y Bridges en 1994, que consta de 10 ítems en escala Likert de 5 puntos (siempre, casi siempre, a veces, casi nunca y nunca), 6 ítems pretenden medir la dimensión de optimismo disposicional, en tanto que los otros 4 ítems son distractores o de "relleno" denominados así por el autor. De los 6 ítems, 3 están redactados en sentido positivo (dirección optimista), y 3 en sentido negativo (dirección pesimista), en diferentes estudios el uso de la prueba ha arrojado un Alfa de Cronbach 0.71 y relaciones entre ítem-total entre 0.40 a 0.70 (Laranjeira, 2008). Estudios con población española, muestran que obtuvo un Alfa de 0.75 (Remor et al. 2006), en muestra peruana se obtuvo un Alfa de 0.61 y de 0.68 en muestra brasileña (Bandeira, Bekou, Silva-Lott, Teixeira y Silva-Rocha, 2002). En la adaptación al castellano original, el Alfa fue de 0.78 y la correlación con el LOT original fue de 0.95 (Otero et al. 1998), las propiedades de la versión adaptada al español son muy similares a las del test original, ya que la escala tiene una estructura clara con dos dimensiones (optimismo y pesimismo) y los factores están negativamente correlacionados, además, todos los ítems tienen un aceptable poder discriminativo (apéndice A).

Prueba de optimismo disposicional: para evaluar el optimismo se utiliza este instrumento elaborado por Londoño, Hernández, Alejo y Pulido (2013), que busca determinar la tendencia personal hacia el optimismo disposicional. Consta de 21 ítems que representan situaciones cotidianas de las personas con tres opciones de respuesta: optimista, sesgo optimista y pesimista. Se realizó el proceso de validación del instrumento con una muestra to- tal de $\mathbf{2 0 0}$ adultos alcanzando un nivel de confiabilidad aceptable de 0.75 , que no se mantuvo en el estudio realizado por Gómez y Londoño (2012), en el que la confiabilidad solo alcanzó un nivel moderado bajo de 0.53 , por eso el presente estudio incluye la revisión de los ítems y las opciones de respuesta.

\section{Procedimiento}

En primer lugar se llevó a cabo una nueva revisión de jueces expertos usando el método específico de Angoff Modificado con el cálculo de un promedio de los puntajes dados por los evaluadores expertos respecto de la pertinencia, suficiencia y redacción. Cada experto calificó cada ítem y presentó de manera argumentada el puntaje asignado, para finalmente tomar una decisión acerca de la inclusión del ítem, su redacción y suficiencia; luego se hizo una revisión y ajuste del instrumento respecto a la redacción de los ítems y las opciones de respuesta de acuerdo con las recomendaciones de los jurados. En un segundo momento, previa firma del consentimiento informado, se aplicó el instrumento a un grupo de 20 personas para evaluar la validez de presentación y se hizo la aplicación de los instrumentos a la muestra seleccionada. En tercera instancia se llevó a cabo el análisis de resultados con el programa Winstep desde la TRI. El análisis y ajuste de datos al modelo se hizo por medio de la estimación del parámetro bajo el método de máxima verosimilitud del Modelo de Rash, que permite identificar un patrón de respuesta observado en los datos.

\section{Resultados}

En los análisis iniciales del instrumento de optimismo disposicional elaborado por Londoño, Hernández, Alejo y Pulido (2013), en el nivel de acuerdo alcanzado por los jueces se determinó la existencia de unanimidad en la calificación por encima de 4 de la pertinencia (promedio=4.7) y de la suficiencia (promedio $=4.33$ ); sobre la redacción se encuentran recomendaciones de precisión del idioma y ajuste en la extensión de los enunciados, que en todos los casos fueron aplicadas (se acorta 
la extensión de los ítems $2,8,14$ y 20 , y se reorganizan las opciones de respuesta para que no se den en un orden constante, ya que siempre aparecían 1) opción pesimismo, 2) opción sesgo optimista no realista y 3 ) opción optimismo disposicional)).

Respecto de la validez de presentación, es posible afirmar que la prueba es de fácil comprensión, teniendo en cuenta que ningún participante expresó dificultad para entender las instrucciones y las opciones de respuesta. Sin embargo, al observar una constante en la elección de la opción de respuesta correspondiente a optimismo disposicional, se decide hacer una aplicación de los ítems como preguntas abiertas, para posibilitar que la alter-

Tabla1.

Resultados análisis de categoría por respuesta.

1. ME INSCRIBO PARA CONCURSAR EN UNA BECA Y PIERDO

1. No me esforcé lo suficiente

2. Tendré otra oportunidad para demostrar mi talento

3. Debo seguir intentándolo

4. No era para mi

5. No importa, no me afecta

2. CUANDO REALIZO TRABAJOS EN GRUPO

1. Tengo que dar lo mejor de mi

2. Es más sencillo y todos colaboran

3. Es difícil, no todos participan activamente

4. Es mejor estar solo

3. GUARDO UNA COPIA CUANDO REALIZO UN TRABAJO

1. Es mejor ser precavido

2. No es necesario hacer esto

3. Siempre guardo una copia por seguridad

4. Me sirve para repaso

4. TENÍA GUARDADO UN TRABAJO IMPORTANTE Y NO LO PUDE IMPRIMIR

1. Debo buscar otra alternativa

2. No es mi día

3. Sacaré una mala nota

4. Debo ser más organizado(a)

5. Es mejor tenerlo en el correo siempre nativa dada fuera libre y así lograr identificar si existía una opción de respuesta que representara la tendencia normal de pensamiento de las personas diferente a las opciones ya establecidas. Para ello se aplica la prueba a 187 personas llevando un registro literal de las opciones dadas para luego realizar un análisis de categorías de respuesta. En la tabla 1 se observa que las contestaciones podrían ser clasificadas en las mismas opciones de respuesta previamente definidas para la prueba; especialmente en la opción correspondiente a optimismo disposicional. No obstante se detecta un posible sesgo educacional en las situaciones presentadas en algunos de los ítems, que merece ser revisado.
5. ME PROPONEN TENER RELACIONES SEXUALES
1. Es algo normal
2. Es interesante
3. Me están irrespetando
4. Depende de quién sea
5. Sí, con protección

6. ME TOMÉ UNOS TRAGOS E HICE EL RIDÍCULO DELANTE DE MIS AMIGOS

1. No les voy a volver a dar la cara

2. Me daría vergüenza

3. Asumiría las consecuencias

4. Ofrecería disculpas

7. CON FRECUENCIA ME INVITAN A JUGAR PLAYSTATION

1. Es una pérdida de tiempo

2. No me gusta

3. Es un buen pasatiempo, aceptaría inmediatamente

4. Se puede volver una adicción

5. Chévere jugar y hacer torneos o apuestas

8. EN UN ALMACÉN LE DAN BONOS DE DESCUENTOS A TODOS MENOS A MÍ

1. Es muy injusto

2. No alcanzaron

3. No soy de su agrado

4. No tuve suerte

5. Son solo para clientes frecuentes 
9. MI PAREJA ME HACE UN REGALO POCO USUAL Y SIN RAZÓN APARENTE

1. Solo quería tener un bonito detalle

2. Hay algún interés oculto

3. Me quiere

4. Quería sorprenderme

10. MI MÉDICO ME DICE QUE CONSUMO DEMASIADA GRASA

1. Me estoy descuidando con mi salud

2. Debo cambiar mis hábitos alimenticios

3. Sigo los consejos del médico

4. No cambio mi estilo de vida, eso no es importante

11. ME CAIGO CON FRECUENCIA MIENTRAS PRACTICO ALGÚN DEPORTE

1. Soy algo torpe, debo tener un problema óseo

2. Es la falta de práctica

3. No soy hábil para practicar deportes

4. Debo buscar ayuda médica

5. A todos nos pasa

12. ME ENTREGAN UNA MALA CALIFICACIÓN EN UNA MATERIA

1. No estudié lo suficiente

2. Debo esforzarme más

3. No entendí muy bien

13. PRESENTO UNA SUGERENCIA A MI GRUPO Y NO ME P'RESTAN ATENCIÓN

1. No soy demasiado importante

2. Debo hablar más fuerte

3. Mis ideas siempre son tenidas en cuenta

4. Estaban distraídos y no me escucharon

14. CONSTANTEMENTE LAS PERSONAS ME DICEN QUE TENGO APARIENCIA DE ESTAR ENFERMO

1. No debo verme nada bien

2. Es mi apariencia natural

3. Debe ser el cansancio

4. Debo ir al médico

Para llevar a cabo la validez concurrente de la prueba, la muestra más o menos equilibrada por género y nivel educativo, tenían una edad promedio de 23.33 , con una DE de 8.33 y reportó que
15. TIMBRA EL TELÉFONO A MEDIA NOCHE

1. Algo malo sucedió

2. Debe ser un número equivocado

3. Es una broma, no contesto

4. Me llaman para invitarme a rumbear

16. SE ME VIENE LA SANGRE POR LA NARIZ

1. Se me rompió un vasito

2. Me estoy enfermando

3. Es normal, no hay de qué preocuparse

17. EL COLECTIVO EN QUE ME TRANSPORTABA SE VARÓ

1. Preciso tenía que pasarme a mi

2. Tomar otro para no llegar tarde a mi trabajo o estudio

3. Tranquilizarme, es normal que pase, así que debo salir más temprano

18. UN AMIGO ME PIDE QUE COMPRE UNA BOLETA DE UNA RIFA

1. Me la podría ganar

2. Quizás no me la gane pero le ayudaré

3. Solo la compro y ya

4. Mi amigo necesita dinero para algo importante

19. DEBO EXPONER SOBRE UN TEMA QUE NO MANEJO

1. Quizás me den más tiempo para prepararla

2. Voy a hacer el ridículo

3. Voy a improvisar

4. Si no estoy preparado es mejor no presentar la exposición

20. SALÍ DISTRAIDO DE MI CASA Y OLVIDÉ QUE ES PICO Y PLACA

1. Seguro me van a colocar un parte

2. ¡Qué tonto soy, cómo pude olvidarlo!

3. Quizás si me devuelvo no haya problema

21. UN AMIGO ME PROPONE QUE MONTEMOS UN NEGOCIO DE COMIDAS RÁPIDAS

1. Sería una gran idea

2. Un negocio propio es algo bueno

3. Las sociedades no son buenas, siempre terminan mal

la mayoría de ellos hacen parte de una religión (75.7\%), pero menos de la mitad de estos participantes realiza alguna práctica religiosa $(20.4 \%)$ (tabla 2). 
Tabla 2.

Información sociodemográfica de los participantes.

\begin{tabular}{|c|c|c|c|c|c|c|c|}
\hline & $\mathrm{F}$ & $\%$ & & & & $\mathrm{~F}$ & $\%$ \\
\hline Sexo & & & & & Nivel educativo & & \\
\hline Mujeres & 336 & 55.3 & & & Bachiller & 182 & 29.9 \\
\hline Hombres & 272 & 44.7 & & & Técnico & 167 & 27.5 \\
\hline Estrato & & & & & Profesional & 147 & 24.2 \\
\hline 0 & 4 & 0.7 & & & Postrados & 112 & 18.4 \\
\hline 1 & 99 & 16.3 & & & Religión & & \\
\hline 2 & 236 & 38.8 & & & Si & 460 & 75.7 \\
\hline 3 & 220 & 36.2 & & & No & 147 & 24 \\
\hline 4 & 33 & 7.1 & & & no responde & 1 & 0.2 \\
\hline \multirow[t]{4}{*}{5} & 6 & 1 & & & práctica religiosa & & \\
\hline & & & & & $\mathrm{Si}$ & 124 & 20.4 \\
\hline & & & & & No & 483 & 79.4 \\
\hline & $x$ & $\mathrm{De}$ & Min & $\operatorname{Max}$ & & & \\
\hline Edad & 23.33 & 8.33 & 16 & 60 & & & \\
\hline
\end{tabular}

Respecto de la confiabilidad, se evidencia que desde la TRI el cuestionario cuenta con un buen nivel, aunque el Alpha de Cronbach que surge del análisis de la Teoría Clásica de los Test es considerado bajo. Los errores de medida se aproximan a cero, ello indica que la medida tiende a ser exacta (tabla 3).

Tabla 3.

Resumen de las medidas y cálculos de los índices de confiabilidad del cuestionario de optimismo.

\begin{tabular}{lcccccccc}
\hline & $\begin{array}{c}\text { Puntaje } \\
\text { total }\end{array}$ & $\begin{array}{c}\text { Conteo } \\
\text { de casos }\end{array}$ & Medida & $\begin{array}{c}\text { Error del } \\
\text { modelo }\end{array}$ & MNSQ & ZSTD & MNSQ & ZUTFIT \\
MEDIA & 25.0 & 22.0 & 0.36 & 0.36 & 0.98 & -0.3 & 1.00 & -0.3 \\
D.T. & 3.6 & .5 & 0.47 & 0.02 & 0.57 & 2.0 & 0.62 & 2.0 \\
Max. & 34.0 & 22.0 & 1.70 & 0.73 & 2.91 & 4.3 & 3.37 & 4.2 \\
Min. & 2.0 & 12.0 & -3.48 & 0.33 & 0.13 & -4.7 & 0.12 & -4.9 \\
REAL RMSE & 0.40 & de real 0.25 & & Separación 0.62 & & Confiabilidad 0.28 & \\
MODEL RMSE .3656 & de real 0.30 & Separación 0.84 & & Confiabilidad 0.41 & \\
De participantes & 0.02 & & & & & & \\
\hline
\end{tabular}

Notas: DT: Desviación típica; MNSQ: Mínimos Cuadrados Normados; RSME: Raíz Cuadrada del Error Cuadrático Medio

Al analizar los infit para detectar la existencia de respuestas anómalas y el outfit para detectar comportamientos anómalos que estén lejos del puntaje general de cada sujeto; es posible evidenciar el buen funcionamiento de los ítems 5, $7,10,11$ a 18,20 y 22 , ya que en todos los casos los puntajes van desde 1 hasta 1.3 , ello indica que tienen un nivel óptimo de ajuste. Para los ítems $1,2,3,4,6,8,9,19$ y 21 podrían considerarse sobre-ajustados; pero al tener en cuenta que en los outfit que sean menores de 2.0 , se considera que todos los ítems están correctamente ajustados al modelo de Rash (tabla 4). 


\begin{tabular}{|c|c|c|c|c|c|c|c|c|c|c|c|c|c|c|c|c|}
\hline \multirow{2}{*}{ 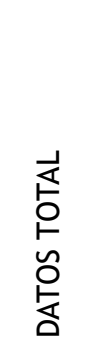 } & \multirow{2}{*}{ 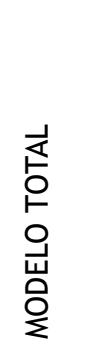 } & \multirow{2}{*}{ 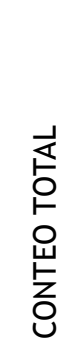 } & \multicolumn{2}{|r|}{$\begin{array}{l}\text { 옴 } \\
\text { 을 }\end{array}$} & \multicolumn{2}{|c|}{ 贞 } & \multicolumn{2}{|c|}{$\begin{array}{l}\text { 点 } \\
\frac{5}{5}\end{array}$} & \multicolumn{2}{|c|}{ 志 } & \multicolumn{2}{|c|}{ 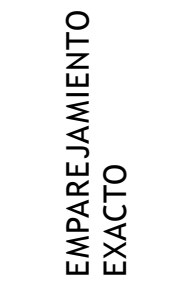 } & \multirow{2}{*}{ 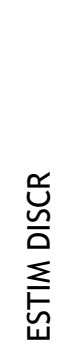 } & \multirow{2}{*}{ 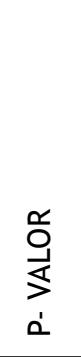 } & & \multirow[b]{2}{*}{ ט } \\
\hline & & & MEASURE & S.E. & MNSQ & ZSTD & MNSQ & ZSTD & CORR. & EXP. & OBS\% & EXP\% & & & & \\
\hline 1 & 659 & 615 & .0332 & .0858 & .98 & -.3 & .98 & -.31 & .26 & .22 & 76.4 & 76.4 & 1.02 & 1.07 & 001 & 0 \\
\hline 2 & 825 & 616 & -.4210 & .0638 & .99 & -.31 & .97 & -.6 & .31 & .29 & 47.7 & 49.0 & 1.03 & 1.34 & 002 & 0 \\
\hline 3 & 983 & 616 & -.7638 & .0651 & .93 & -1.0 & .92 & -1.1 & .37 & .30 & 65.4 & 64.8 & 1.05 & 1.60 & 003 & 0 \\
\hline 4 & 766 & 613 & -.2402 & .0641 & .96 & .9 & .96 & -.9 & .35 & .28 & 51.5 & 52.3 & 1.08 & 1.25 & 004 & 0 \\
\hline 5 & 585 & 614 & .5511 & .0809 & 1.03 & .5 & 1.02 & .3 & .17 & .23 & 73.8 & 73.7 & .97 & .95 & 005 & 0 \\
\hline 6 & 673 & 616 & .0585 & .0731 & .99 & -.2 & .99 & -.2 & .27 & .25 & 67.0 & 66.9 & 1.02 & 1.09 & 006 & 0 \\
\hline 7 & 550 & 616 & .6541 & .0663 & 1.11 & 2.2 & 1.12 & 2.4 & .08 & .26 & 59.6 & 59.5 & .82 & .89 & 007 & 0 \\
\hline 8 & 816 & 615 & -.4558 & .0660 & .97 & -.6 & .96 & -.7 & .32 & .28 & 53.7 & 52.0 & 1.05 & 1.33 & 008 & 0 \\
\hline 9 & 1009 & 616 & -.6379 & .0608 & .89 & $-1.6 \mid$ & .81 & -2.2 & .45 & .32 & 73.2 & 68.4 & 1.07 & 1.64 & 009 & 0 \\
\hline 10 & 603 & 616 & .4279 & .0700 & 1.07 & $1.3 \mid$ & 1.14 & 2.5 & .12 & .26 & 64.1 & 64.7 & .90 & .98 & 010 & 0 \\
\hline 11 & 591 & 616 & .5017 & .0745 & 1.00 & .0 & .99 & -.1 & .25 & .24 & 69.2 & 68.9 & 1.00 & .96 & 011 & 0 \\
\hline 12 & 732 & 616 & -1917 & .0695 & 1.01 & .3 & 1.01 & .2 & .24 & .26 & 60.7 & 60.9 & .98 & 1.19 & 012 & 0 \\
\hline 13 & 751 & 613 & -.3091 & .0703 & 1.00 & .0 & .99 & -.1 & .27 & .26 & 59.5 & 60.1 & 1.00 & 1.23 & 013 & 0 \\
\hline 14 & 390 & 611 & -.2286 & .0862 & .97 & -.9 & 1.00 & .0 & .25 & .21 & 67.6 & 65.3 & 1.08 & .64 & 014 & 0 \\
\hline 15 & 530 & 614 & .6934 & .0626 & 1.03 & .6 & 1.05 & 1.0 & .23 & .27 & 53.4 & 54.0 & .94 & .86 & 015 & 0 \\
\hline 16 & 649 & 614 & .2183 & .0651 & 1.02 & .4 & 1.02 & .3 & .25 & .27 & 58.3 & 58.5 & .97 & 1.06 & 016 & 0 \\
\hline 17 & 667 & 613 & .1582 & .0624 & 1.00 & .0 & 1.00 & .0 & .29 & .28 & 54.6 & 54.2 & 1.00 & 1.09 & 017 & 0 \\
\hline 18 & 970 & 615 & -.4888 & .0566 & 1.02 & .3 & 1.13 & 1.5 & .30 & .33 & 64.9 & 62.9 & .98 & $\begin{array}{c}1 \\
1.58\end{array}$ & 018 & 0 \\
\hline 19 & 672 & 615 & .1375 & .0635 & .92 & -1.8 & .91 & -1.8 & .41 & .28 & 56.9 & 55.8 & 1.17 & 1.09 & 019 & 0 \\
\hline 20 & 648 & 615 & .1197 & .0851 & 1.03 & .5 & 1.03 & .4 & .15 & .22 & 76.1 & 76.2 & .97 & 1.05 & 020 & 0 \\
\hline 21 & 653 & 615 & 1371 & .0769 & .98 & -.3 & .98 & -.3 & .28 & .24 & 70.4 & 70.6 & 1.02 & 1.06 & 021 & 0 \\
\hline 22 & 675 & 613 & .0459 & .0718 & 1.04 & $.8 \mid$ & 1.04 & .8 & .18 & .25 & 65.4 & 65.3 & .95 & 1.10 & 022 & 0 \\
\hline MEDIA & 699.9 & 614.7 & .0000 & .0700 & 1.00 & -.1 & 1.00 & .0 & & & 63.2 & 62.7 & & & & \\
\hline DT & 147.7 & 1.4 & .4065 & .0083 & .05 & .9 & .07 & 1.1 & & & 8.0 & 7.7 & & & & \\
\hline
\end{tabular}

En este caso los puntajes brutos de los reactivos se ubican en una sola dirección, es decir que el constructo es unidimensional; sin embargo se pre- sentan 4 subconjuntos de ítems o características dentro del constructo general. 
Figura 1.

Diagrama de distribución del Cuestionario de Optimismo Disposicional revisado.

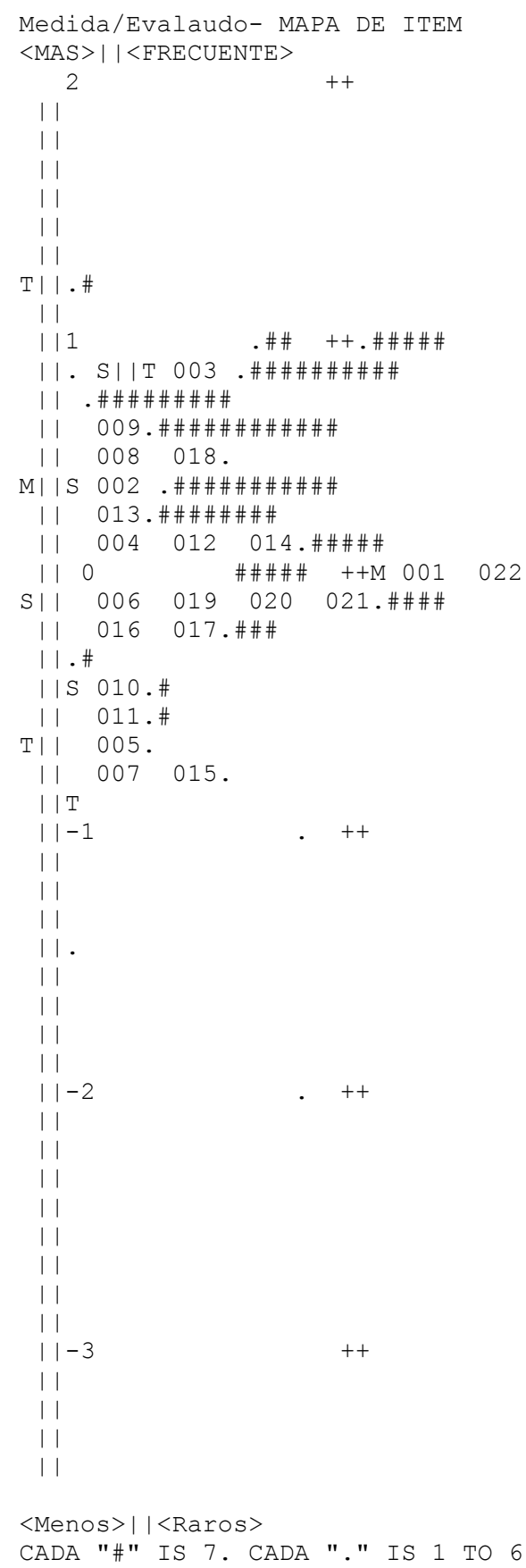

El primero que recoge los ítems $3,9,8$ y 18 , que parecen discriminar alto optimismo o sesgo optimista no realista; los ítems 2, 13, 12, 4 y 14 que recogen a los individuos con pensamiento optimista disposicional, los ítems $1,6,16,17,20,21,22$ y 19 que recogen a los individuos con un pensamiento normal, y los ítems que identifican especialmente a los pesimistas $10,11,5,7$ y 15 (figura 1 ). 
La distribución en la figura 1 que representa la ubicación de los ítems y los participantes se encuentran entre 3 y -1 logits de forma casi paralela, lo que indica que existe alta comprensión semántica de quienes responden a los ítems y el grado en el que se presenta el constructo; es decir que la relación entre los mismos denota que estos son discriminativos respecto de los puntajes reportados en la prueba.

Para obtener la forma de calificación se transforman los puntajes en medidas más amplias para sensibilizar los estadísticos y reconocer los diferentes grupos clasificados en la prueba. Con las nuevas puntuaciones, para la calificación de la escala, se tiene en cuenta la media obtenida en el modelo y la desviación estándar, que representan las medidas base para definir los puntos de corte. Por cada respuesta de optimismo dada, si la opción pertenece a sesgo optimista no realista indica que se otorga una puntuación de 10; si es de optimismo de 5 y si es de pesimismo es de 1 punto; con una calificación total máxima posible de 220 y mínima de 22 .

La media obtenida por la población fue de 129, con una desviación estándar 16 , con un puntaje mínimo de 31 y uno máximo 171; el optimista dis- posicional puntuaría típicamente en el rango comprendido entre la media más uno y una desviación estándar $(X+1$ y +1 de), un optimista con sesgo no realista se ubicaría por encima de una desviación estándar (+1 de +1$)$, y el pesimismo estaría por debajo de la media a 1 desviación estándar, es decir $(X-1$ de $=$ pesimista). Traducido en términos de puntuaciones por cada factor, quiere decir que puntajes entre 130 y 146 recogen a las personas con optimismo disposicional, los puntajes inferiores a 113 indican pesimismo, y los puntajes por encima de 147 indican la presencia de sesgo.

Al analizar el LOT bajo los parámetros del modelo de Rash, se evidencia un bajo nivel de confiabilidad y problemas en los ítems correspondientes a optimismo; no obstante tomando los indicadores de la TCT de confiabilidad, se realizan los análisis de correlación entre los puntajes de optimismo obtenidos en ambas escalas y entre los de pesimismo de la misma forma. En la tabla 5 , se encuentra que existe correlación directa y significativa entre los factores de pesimismo medidos con los instrumentos utilizados, e indirecta entre el factor optimismo del COD y el factor pesimismo del LOT. No obstante, no se encuentran correlaciones significativas entre el factor optimismo del LOT y el factor optimismo del COD.

Tabla 5.

Correlación de las Pruebas.

\begin{tabular}{lcccc}
\hline & COD optimismo & COD pesimismo & LOT optimismo & LOT pesimismo \\
\hline COD optimismo & 1 & $-0.380^{* *}$ & $0.138^{*}$ & $0.276^{* *}$ \\
COD pesimismo & & 1 & $-0.182^{* *}$ & $0.386^{* *}$ \\
LOT optimismo & & & 1 & $0.216^{* *}$ \\
LOT pesimismo & & & & 1 \\
\hline
\end{tabular}

Notas: ${ }^{* *}$ p menor o igual a $0.001,{ }^{*} p$ menor o igual a 0.05

Se incluye la ficha técnica del COD, para facilitar la identificación de las características finales de la prueba, ya que explica los criterios relacionados con este tipo de instrumentos, dando a conocer algunas generalidades importantes para su posterior aplicación. 
Tabla 6.

Ficha técnica del Cuestionario de Optimismo Disposicional.

\begin{tabular}{ll}
\hline Aspecto & Descripción \\
\hline Características generales & $\begin{array}{l}\text { El Cuestionario de Optimismo Disposicional está compuesto por xx ítems de fácil com- } \\
\text { presión, que están relacionados con el optimismo/ pesimismo. }\end{array}$ \\
Opciones de respuesta & $\begin{array}{l}\text { Son frases que describen pensamientos y conductas típicas de individuos optimistas } \\
\text { disposicionales, con sesgo optimista no realista y pesimistas }\end{array}$ \\
Administración & Individual o Grupal \\
Duración & Tiempo promedio 15 minutos a 20 minutos; sin límite de tiempo \\
Dirigido & Personas entre xx y xx años \\
Objetivo & Evaluar presencia de estilo optimista disposicional en jóvenes y adultos \\
Normas & $\begin{array}{l}\text { Realizar el cuestionario sin ningún tipo de colaboración } \\
\text { Responder a todos los ítems en una misma sesión } \\
\text { Evitar cambiar la respuesta }\end{array}$ \\
& $\begin{array}{l}\text { Cada opción de respuesta se puntúa de la siguiente forma: } \\
\text { Sesgo optimista no realista indica una puntuación de 10 en cada ítem contestado de } \\
\text { esa forma } \\
\text { Optimismo una puntuación de } 5 \\
\text { Pesimismo de 1 punto }\end{array}$ \\
Puntuación & La calificación se obtiene con la suma de las puntuaciones obtenidas en cada ítem \\
& $\begin{array}{l}\text { Calificación: (p1 + p2 +... P22) } \\
\text { La puntuación total de los ítems está por cortes distribuidos así: } \\
\text { Entre 130 y 146: optimismo disposicional } \\
\text { Menos de 114: pesimismo } \\
\text { Más de 147: sesgo optimista no realista }\end{array}$ \\
\hline Interpretación &
\end{tabular}

Los ítems definitivos del COD se presentan en el apéndice A para facilitar la comprensión de la discusión y las conclusiones. En general los ítems que conforman el cuestionario cumplen con los parámetros establecidos para su diseño, ya que identifican la forma de pensar y actuar de personas que tienen un estilo optimista disposicional.

\section{Discusión y conclusiones}

Ya que el objetivo de esta investigación era diseñar y evaluar las características psicométricas (validez y confiabilidad) del Cuestionario de Optimismo Disposicional -COD- para jóvenes y adultos, se tomó una muestra equilibrada por género, de estratos 0 al 5 , solteros y de distintos niveles de educación,
Para determinar la calidad psicométrica del COD se llevaron a cabo los análisis desde la TRI, pues facilita una medición conjunta, es decir analiza las interacciones entre las personas y los ítems, ello permitió determinar que el Cuestionario tiene alta confiabilidad y calidad psicométrica, teniendo en cuenta que aunque el Alpha de Cronbach no es elevado pero el nivel de separación reportado desde el modelo de Rasch es bajo, es decir óptimo, esto indica un nivel adecuado de confiabilidad; cabe anotar que las medidas obtenidas con el Modelo de Rasch son consideradas psicométricamente más robustas que las obtenidas con la TCT (Prieto y Delgado, 2003).

Además el COD tiene alta consistencia interna que combinada con el nivel de separación reportado 
indican un nivel de ajuste apropiado; lo cual permite pensar que se está evaluando el constructo objetivo de esta investigación, determinando la presencia o ausencia de la tendencia cognitiva y comportamental a ser optimistas. Esto teniendo en cuenta que los índices de separación que según la TRI indican que los respondientes comprendieron los ítems y que su respuesta refleja lo que deseaban contestar (González, 2008); condición que se reconfirma al observar la alineación entre el comportamiento de los ítems y el de los participantes (figura 1); es decir, que las medidas obtenidas con la prueba son relativamente independientes a las condiciones en las que son realizadas las aplicaciones y que por este motivo ningún ítem fue excluido.

Los ítems que estaban dentro del rango permitieron establecer el cumplimiento del principio de independencia entre los ítems, es decir que cada respuesta dada es independiente de las otras dadas a cada pregunta, indicando que el instrumento es consistente y tiende a ser discriminativo frente a las presencia o ausencia de un estilo optimista disposicional de respuesta. De otra parte, la totalidad de los ítems muestran un ajuste moderado que indica que las diversificaciones en las respuestas se imputan a las diferencias individuales y a los ítems redactados para medir el constructo (Prieto y Delgado, 2003), esto indica que la prueba puede ser usada para medir el optimismo disposicional.

Los resultados muestran que todos los ítems están relacionados con el constructo previamente definido, esto implica que se logrará mayor precisión en la medición del Optimismo Disposicional utilizando el COD; con una agrupación en cuatro subconjuntos de ítems que resultan cualitativamente relacionados con los cuatro estilos descritos en la literatura y tomados como base para la construcción del cuestionario: optimismo disposicional ( 2 , $13,12,4$ y 14$)$, sesgo optimista no realista $(3,8$, 9 y 18), pensamiento normal $(1,6,16,17,19,20$, 21 y 22$)$ y pesimismo $(5,7,10,11$, y 15$)$. Distribución que indica que quienes elijan en la opción de respuesta la correspondiente al factor al que pertenece cada uno de estos ítems, tenderá a ubicarse en el puntaje total ubicado en esa categoría general (Prieto y Delgado, 2003).
La versión final del instrumento quedó similar a la primera versión, salvo el ajuste a la pregunta 21 (un amigo me propone que montemos un negocio de comidas rápidas), que fue modificada debido a que la situación descrita no correspondía a un evento general que pudiera se imaginado por cualquier individuo que respondiera la prueba. Cabe anotar que entre 0.5 y 1.5 se considera que la situación es productiva, es decir que las variaciones en los puntajes no son debidas al azar sino a cambios en el cumplimiento de la condición o constructo (González, 2008).

Respecto del análisis de la validez concurrente, es posible indicar que la baja confiabilidad encontrada desde la TRI del LOT, hace que sea necesario conducir nuevos estudios en los que se analicen las condiciones psicométricas del instrumento con poblaciones diferentes. Sin embargo, ya que el LOT por su amplio uso ha sido tomado como medida probablemente fiable, se analiza que las correlaciones indican la existencia de cierta concordancia entre las medidas obtenidas de pesimismo en cada una de los instrumentos, y una asociación inversa entre el factor optimismo medido por el LOT y el de optimismo medido con el COD; esto podría tomarse como indicio de que el factor optimismo es linealmente opuesto con el factor pesimismo tal como lo propone el COD, pero que las dificultades psicométricas del LOT puedan estar afectando los resultados; e incluso sería necesario conducir estudios cualitativos de análisis de contenido de entrevistas que permitan corroborar los resultados obtenidos por las pruebas. De otra parte, la extensión claramente disímil de las pruebas, puede afectar la capacidad discriminativa de las mismas, ya que la suficiencia de los ítems para medir un estilo global permanente como el del optimismo o del pesimismo puede verse limitada debido a que en el LOT solo se cuenta con 6 preguntas para definir un estilo de pensamiento y comportamiento que se espera sea estable en diversas situaciones. Finalmente es posible concluir que el COD fue aceptado favorablemente por los participantes de la investigación, puesto que la totalidad de los ítems presentan condiciones que cumplen con los parámetros del Modelo Rasch y en general la prueba mide de manera óptima el Optimismo Disposicional. 
Entre las limitaciones del estudio se encuentra el hecho de no contar con una segunda medida de optimismo/pesimismo más robusta o de entrevistas reconfirmatorias, y se denota la existencia de un posible sesgo educacional por el tipo de situaciones incluidas en los ítems, por ello se pretenden realizar nuevos estudios en este sentido. Asimismo, todavía queda un camino largo por recorrer en el desarrollo de medidas que faciliten la identificación rápida y oportuna de estilos cognitivos y comportamentales de los individuos que resultan favorables a su calidad de vida, desempeño social, laboral y académico y que faciliten el afrontamiento de situaciones que le resulten estresantes al individuo.

\section{Referencias}

Abrahamson, L., Seligman, Y., y Teasdale, M. (1978). Learned helplessness in humans: Critique and Reformulation. Abnormal Psychology. 87, 49-74. Recuperado de http://psyc net.apa.org/index.cfm?fa=buy.optionTo Buy\&id $=1979-00305-001$

Alpízar, H., y Salas, D. (2010). El papel de las emociones positivas en el desarrollo de la Psicología Positiva. Revista Electrónica de estudiantes Esc. de psicología, 5(1), 65-83. Universidad de Costa Rica. Recuperado de http://www. dialnet.unirioja.es

Anadón-Revuelta, O. (2006). Inteligencia emocional percibida y optimismo disposicional en estudiantes universitarios. [Versión electrónica]. Revista Electrónica Interuniversitaria de Formación del Profesorado, 9(1), 1-13. Recuperado de http://www.aufop.com/aufop/ uploaded_files/articulos/1224455027.pdf

Andrews, F. M., y Withey, S. B. (1976). Social indicators of well-being: The development and measurement of perceptual indicators. New York: Plenum.

Bandeira, M., Bekou, V., Silva Lott, K., Teixeira, M., y Silva Rocha, S. (2002). Validação transcultural do Teste de Orientação da Vida (TOVR). Estudos de Psicologia, 7, 251-258. Recuperado de http://www.scielo.br/pdf/epsic/v7n2 /a06v07n2.pdf
Begoña, M., Muñoz, R., Panduro, M., Crespillo, Y., Rojas, L., y González, S. (2010). El proceso de afrontamiento en personas recientemente ostomizadas. Index de Enfermería, 19(2-3), 115-119. Recuperado de http://scielo.isciii.es/ scielo.php?pid=S1132-12962010000200009

Black, J., y Reynolds, W. (2013). Examining the relationship of perfectionism, depression, and optimism: Testing for mediation and moderation. Personality and Individual Differences, 54(3), 426-431. DOI: 10.1016/j.paid.2012. 10.012.

Brown G. F. (1999). Principios de la medición en psicología y educación. México: Manual Moderno.

Chico, E. (2002). Optimismo disposicional como predictor de estrategias de afrontamiento. Psicothema, 14(3), 544-550. Recuperado de http://dialnet.unirioja.es/servlet/articulo?co digo=1103085\&orden=33104\&info=link

Cuadra, H., y Florenzano, R. (2003). El bienestar subjetivo: hacia una psicología positiva. Revista de psicología de la Universidad de Chile, 12(1), 83-96. Recuperado de http://re dalyc.uaemex.mx/src/inicio/ArtPdfRed.jsp? iCve $=26400105$

Diener, E., Suh, E., Lucas, R., y Smith, H. (1999). Subjective well-being: the decades of progress. Psychological Bullerin. 125(2), 276-302. Recuperado de http://internal.psychology. illinois.edu/ ediener/Documents/Diener-SuhLucas-Smith_1999.pdf

Diener, E., y Suh, E. M. (2000). Culture and Subjective Well-Being. Cambridge: MIT Press.

Gison, A., Dall'armi, V., Donati, V., Rizza, F., y Giaquinto, S. (2014). Dispositional optimism and Parkinson's disease. Annals of Physical and Rehabilitation Medicine, 57(1), 364. DOI: 10.11138/FNeur/2014.29.1.005.

Gómez, C., y Londoño, C. (2013). Modelo predictor del consumo responsable de alcohol y el comportamiento típicamente no violento en adolescentes. Salud y Drogas, 13(1), 2333. Recuperado de www.redalyc.org/pdf/ 839/83928046003.pdf 
González, M. (2008). Análisis de reactivos con el modelo Rasch. Instituto para la medición de la educación, 2(2), 1-110.

Hartmann, U., Chneider, U., y Emrich, M. (2003). La búsqueda de la felicidad. Revista Mente y $\mathrm{Ce}$ rebro, 4, 78-83. Recuperado de http://dialnet. unirioja.es/servlet/articulo?codigo $=2809082$

Heinonen, K., Raikkonen, K., Matthews, K. A., Scheier, M. F., Raitakari, O. T., Pulkki, L., y Keltikangas-Jarvinen, L. (2006). Socioeconomic status in childhood and adulthood: Associations with dispositional optimism and pessimism over a 21- year follow-up. Journal of Personality, 74, 1.112-1.126. DOI: 10.1111/j. 1467-6494.2006.00404.x.

Naghi, J., Philip, A., Cleenewerck, L., y Schwarz, R. (2012). The effects of spiritualy and religion on outcomes in patients with chronic heart failure. Journal of religion and health. 51(4), 1.124-1.136. PMID: 23304705.

Ji, L., Zhang, Z., Usborne, E., y Guan, Y. (2004). Optimism across cultures: In response to the severe acute respiratory syndrome outbreak. Asian Journal of Social Psychology, 7, 25-34. DOI: 10.1111/j.1467-839X.2004.00132.x.

Kennedy, H., y Hughes, L: (2004). The optimismneuroticism question: an evaluation based on cardiovascular reactivity in female college students. The Psychological Record, Gambier, 54(3), 373. Recuperado de http:// www.scielo.org.co/scieloOrg/php/reflinks. php? refpid $=$ S0123-91552009000100009000 19\&lng=en\&pid=S0123-915520090 00100009

Lai, L., y Yue, X. (2000). Measuring optimism in Hong Kong and mainland Chinese with the revised Life Orientation Test. Personality and Individual Differences, 28, 781-796. DOI: http:// dx.doi.org/10.1016/S0191-8869(99)00138-5

Laranjeira, C. A. (2008). Tradução e validação portuguesa do revised life orientation test (LOT-R). Universitas Psychologica, 7, 469-476. Recuperado de http://www.scielo.org.co/ pdf/rups/v7n2/v7n2a13.pdf
Londoño, C., Alejo, I., Velasco, M., y Botero, P. (2013). Qué nos hace optimistas (en proceso de publicación).

Londoño, C., Hernández, L., Alejo, I., y Pulido, D. (2013). Diseño y validación de la Escala de Optimismo Disposicional/Pesimismo -EOP-. Universitas Psychologica, 1(12), 139-155. Recuperado de http://revistas.javeriana.edu. co/sitio/psychologica/sccs/articulo.php?id=70 1\&PHPSESSID=b40951e99064c3897f615e54a9 a950e0

Londoño, C. (2009). Optimismo y salud positiva como predictores de la adaptación académica. Acta colombiana de psicología: 12(1), 95-107. Universidad Católica de Colombia. Recuperado de http://portalweb.ucatolica.edu. co/easyWeb2/files/23_3051_v12n1-art8.pdf

Marrero. R., y Carballeira, M. (2010). El papel del optimismo y del apoyo social en el bienestar subjetivo. Salud mental, 33, 39-46. Recuperado de http://www.artemisaenlinea.org. mx/acervo/pdf/salud_mental/El\%20papel\%20 del\%20optimismo.pdf

Morales, A. I., Arenas, M. D., Reig-Ferrer, A., Álvarez, F., Malek, T., Moledous, A., Gil, M. T., y Cotilla, E. M. (2011). Optimismo disposicional en pacientes en hemodiálisis y su influencia en el curso de la enfermedad. Nefrología, 31(2), 199-205. Recuperado de http://dps. ua.es/es/documentos/pdf/2011/optimismodisposicional-pdf.

Oreskovic, N., y Goodman, E. (2013). Association of optimism with cardiometabolic risk in adolescents. Journal of Adolescent Health, 52(4), 407-412. DOI: 10.1016/j.jadohealth.2012.09.011.

Ortiz, J., Ramos, N., y Vera-Villarroes, P. (2003). Optimismo y salud: estado actual e implicaciones para la psicología clínica y de la salud. Suma psicológica, 10, 119-134. Recuperado de http://www.academia.edu/560153/ Optimismo_y_Salud_Estado_actual_e_implicaciones_para_la_psicologia_clinica_y_de_ la_salud 
Pere, J., Chico, E., y Tous, J. (2002). Propiedades psicométricas del test de optimismo Life Orientation Test. Psicothema, 1(3), 673-680. Recuperado de http://www.psicothema.com/ pdf/782.pdf

Peterson, C., y Seligman, M. E. P. (1984). Causal explanations as a risk factor for depression: Theory and evidence. Psychological Review, 91, 347-374. Recuperado de http://www. um.es/analesps/v22/v22_1/05-22_1.pdf

Prieto, G., y Delgado, A. (2003). Análisis de un test mediante el modelo de Rasch. Psicothema, 15(1), 94-100. Recuperado de http://www. psicothema.com/pdf/1029.pdf

Remor, E., Amorós, M., y Carrobles, J. (2006). El optimismo y la experiencia de ira en relación con el malestar físico. Anales de Psicología, 22(1), 37-44. Recuperado de http://redalyc. uaemex.mx/redalyc/src/inicio/ArtPdfRed. jsp?iCve $=16722105 \& i C v e N u m=0$

Salas, J. (2008). Control, health and welfare. Suma Psicológica, 15(1). Recuperado de http://redalyc.uaemex.mx/pdf/1342/134212604001.pdf

Sandin, B., Chorot, P., Lostao, L., Joiner, T. Santed, M., y Valiente, R. (1999). Escalas planas de afexto positive y negative: validaciòn facotiral y convergencia transcultural. Psicothema, 11(1), 37-55. Recuperado de http://www. unioviedo.net\%2Freunido\%2Findex.php\%2FP ST\%2Farticle\%2Fdownload\%2F7556\%2F7420\& ei=_FD9UN2EHafp0AG

Scheier, M., Carver, C., y Bridges, M. (1994). Distinguishing optimism from neuroticism (and trait anxiety, self-mastery, and self-esteem): A reevaluation of the Life Orientation Test. Journal of Personality and Social Psychology, 67, 1.063-1.078. Recuperado de http://psycnet.apa.org/psycinfo/1995-07978-001

Scheier, M., y Carver, C. (1985). Optimism, coping and health: Assessment and implications of generalized outcome expectancies. Health Psychology. 4, 219-247.

Scheier, M. F., y Carver, C. S. (2014). Dispositional optimism. Trends in cognitive sciences, 18(6), 293-299. DOI: 10.1371/journal.pone.0097752.

Seligman, M., Duckworth, J., y Steen, T. (2005). Positive psychology in clinical practice. Annual Review, Clinical Psychology. Recuperado de http://arjournals.annualreviews.org/

Senkus, E., Cardoso, F., y Pagan, O. (2014). Time for more optimism in metastatic breast cancer? Cancer treatment reviews, 40(2), 220228. DOI: 10.1016/j.ctrv.2013.09.015.

Tomás, I., González, V., y Gómez. (2000). Teoría de respuesta al ítem y análisis factorial confirmatorio: dos métodos para analizar la equivalencia psicométrica en la traducción de cuestionarios. Psicothema, 12(2), 540-544. Recuperado de http://www.psicothema.com

Velasco, M., y Londoño, C. (2009). Determinantes psicosiales de la permanencia en el programa de reintegración en desmovilizados. Revista Iberoamericana de psicología: Ciencia y Tecnología. 2(2), 17-32. Recuperado de http:// www.iberoamericana.edu.co/Publicaciones/ RevistalberoPsicologia/R03_ARTICULO2_ PSIC.pdf

Vera-Villarroel, P., Córdova-Rubio, N., y Celis-Atenas, K. (2009). Evaluación del optimismo: un análisis preliminar del Life Orientation, test versión revisada (LOT-R) en población chilena. Universitas Psychologica, 8(1), 61-67. Recuperado de http://redalyc.uaemex.mx/ pdf/647/64712168005.pdf

Vera, B. (2006). Psicología Positiva: Una nueva forma de entender la psicología. Papeles del Psicólogo, 27(1), 3-8. Recuperado de http:// redalyc.uaemex.mx/src/inicio/ArtPdfRed. jsp?iCve $=77827102$ 


\section{Apéndice A.}

\section{Cuestionario de Optimismo \\ Disposicional para jóvenes}

El presente cuestionario pretende identificar la forma como usted actúa o piensa en diversas situaciones de la vida diaria; ya que algunas situaciones son imaginadas por favor responda teniendo en cuenta lo que piensa y hace comúnmente, subraye la frase elegida

1. Me inscribo para concursar por una beca y pierdo.
a. Eso se sabía, siempre hay alguien mejor que yo
b Había muchas personas inscritas, habrá una segunda oportunidad
c. Esperaré, de pronto haya alguien que la rechace

2. Cuando realizo trabajos en grupo.
a. Se presentan discusiones interesantes y grandes aportes
b. Mi opinión no cuenta, así que el trabajo lo van a hacer ellos
c. Seguro que me va bien ya que varias ca- bezas piensan más que una

3. Guardo una copia cuando realizo un trabajo.
a. Pienso que no es necesario, nada va a pa- sar
b. Es mejor prevenir que lamentar
c. Soy tan de malas que seguro se me borra

4. Tenía guardado un trabajo importante y no lo pude imprimir.
a. Seguiré intentando, es imposible que se dañe
b. A mí siempre me pasa... y me sigue pa- sando
c. A lo mejor no lo guardé correctamente

5. Me proponen tener relaciones sexuales.
a. Si utilizo un buen método anticonceptivo probablemente no habrá problemas
b. Con una sola vez seguro no tendré proble- mas
c. Si lo hago, seguramente se me pegará al- guna enfermedad

6. Me tomé unos tragos e hice el ridículo delante de mis amigos.

a. Ahora piensan que soy lo peor y no me volverán a invitar a salir

b. Les pido disculpas y pienso que debo controlar mejor estas situaciones
c. A nadie le molesta lo que los borrachos hacen, pronto lo olvidarán

7. Con frecuencia me invitan a jugar PlayStation.
a. Soy el único desocupado que tiene tiempo de jugar con ellos
b. Les gusta perder, pues soy invencible
c. Sé jugar muy bien, además soy muy diver- tido

8. En un almacén les dan bonos de descuento a todos menos a mí.
a. Probablemente en otra oportunidad me ganaré algo
b. Con seguridad me ganaré un premio mu- chísimo mejor
c. Hasta en esas ocasiones las personas me rechazan

9. Mi pareja me hace un regalo poco usual y sin razón aparente.
a. Lo tengo bajo mi dominio
b. Es detallista y me quiere mucho
c. Me está poniendo los cachos y se está sin- tiendo culpable

10. Mi médico me dice que consumo demasiada grasa.
a. Hay gente que sin cuidarse no se enferma
b. De pronto tengo algo grave por no cuidar mi alimentación
c. El cuidado de mi salud depende de mi ali- mentación


11. Me caigo con frecuencia mientras practico algún deporte.
a. Tengo que practicar para mejorar la téc- nica
b. Definitivamente no sirvo para esto
c. Soy realmente bueno y quieren lesionarme

12. Me entregan una mala calificación en una materia.
a. No sirvo para estudiar y por eso me va mal
b. La próxima será mejor, como sea sacaré buena nota
c. Si estudio un poco más voy a tener éxito

13. Presento una sugerencia a mi grupo y no me prestan atención.
a. Están distraídos y no se fijaron en mi co- mentario
b. Digo cosas tan malas que no tienen nin- guna importancia
c. No me escucharon, volveré a expresar mi idea

14. Constantemente las personas me dicen que tengo apariencia de estar enfermo(a).
a. Estoy realmente enfermo(a) y no me había dado cuenta
b. Aunque no me alarmo demasiado, pienso en hacerme un chequeo
c. Nunca me enfermo, no sé qué les pasa

15. Timbra el teléfono a media noche.
a. Voy a contestar para ver qué ocurre
b. Pasó algo malo, a alguien le ocurrió un ac- cidente
c. Qué bueno que alguien piense en mí a esta hora de la noche

16. Se me viene la sangre por la nariz.
a. Esto es el indicio de una grave enferme-
b. No importa lo que haga, tarde o temprano dejaré de sangrar
c. Seguro fue por el cambio de clima, des- cansaré un rato y pronto pasará

17. El colectivo en el que me transportaba se varó. lo contrario pido mi dinero y tomo otro transporte
a. Pregunto si la reparación no demorará de
b. Espero porque estoy seguro de que la re- paración no demorará
c. Pienso que lo único que falta es que tam- bién haya trancón y llegue mucho más tarde

18. Me han puesto una sanción por no pagar la multa de la biblioteca.
a. No pago la multa y estoy seguro que si in- sisto al bibliotecario obtendré el préstamo
b. Pago la multa y espero que el periodo de sanción no sea muy largo
c. Para que pagar si igual me van a sancionar

19. Un amigo me pide que compre una boleta de una rifa.
a. Compro la rifa porque pienso que tengo muy buena suerte y me ganaré el premio
b. Compro la rifa para que mi amigo no piense que soy tacaño, aunque soy de mala suerte
c. Compro la rifa por ayudar a mi amigo, pero sé cuál es mi verdadera posibilidad de ganar

20. Debo exponer sobre un tema que no manejo.

a. Hago una presentación muy elaborada y repaso hasta el final, aunque sé que me va a ir mal

b. Investigo, acudo a varias fuentes y me preparo porque la idea es aprender
c. Investigo en un solo libro porque creo que es suficiente

21. Un amigo me propone que montemos un negocio de comidas rápidas.
a. Analizo la viabilidad del negocio y los be- neficios antes de tomar cualquier decisión
b. Invierto con la certeza de que tendré bue- nas ganancias muy pronto
c. Busco una excusa porque sé que el nego- cio fracasará y además perderé un amigo

